\title{
Maximized Optical Absorption in Ultrathin Films and Its Application to Plasmon-Based Two-Dimensional Photovoltaics
}

\author{
Carl Hägglund, * S. Peter Apell, and Bengt Kasemo \\ Department of Applied Physics, Chalmers University of Technology, SE-412 96 Göteborg, Sweden
}

\begin{abstract}
For ultrathin films of a given material, light absorption is proportional to the film thickness. However, if the optical constants of the film are chosen in an optimal way, light absorption can be high even for extremely thin films and optical path length. We derive the optimal conditions and show how the maximized absorptance depends on film thickness. It is then shown that the optimal situation can be emulated by tuning of the geometric parameters in feasible nanocomposites combining plasmonic materials with semiconductors. Useful design criteria and estimates for the spatial absorption-distribution over the composite materials are provided. On the basis of efficient exchange of oscillator strength between the plasmonic and semiconductor constituents, a high quantum yield for semiconductor absorption can be achieved. The results are far-reaching with particularly promising opportunities for plasmonic solar cells.
\end{abstract}

KEYWORDS Absorption limit, ultrathin films, effective medium, localized surface plasmon resonances, solar cells

A n important challenge for lowering the costs of electricity produced by photovoltaic solar cells is to reduce their thickness without compromising a high performance. In essence, this means that a high fraction of the incident light must be absorbed and converted to longlived excited charge carriers, despite a reduction of the used amount (thickness) of semiconductor material. This challenge has lately stimulated surging activity in the area of plasmonic solar cells. ${ }^{1-6}$ The basic idea is to use surface plasmon resonances in metal nanostructures to achieve a high cross section for light absorption and to exploit the plasmonic excitations for generation of useful charge carriers in a thin layer of solar cell material nearby. The latter process can be stimulated by the enhanced electromagnetic nearfields around the plasmonic nanostructure, or via an enhanced optical path length resulting from favorable far-field effects. Far-field effects can be especially powerful when coupled to optical or surface-guided modes, and their potential has recently been well covered in reviews. ${ }^{7,8}$ Although the more direct near-field effects have also been investigated to some extent with promising results, ${ }^{3,9}$ a dedicated nearfield approach is so far less explored. ${ }^{8}$ It can be argued, based on the Thomas-Reiche-Kuhn sum rule, that the amount of material required to directly absorb most light incident in the spectral range of interest for photovoltaics corresponds to only an around $10 \mathrm{~nm}$ thick film (see the Supporting Information). Considering that typical thin film solar cells have thicknesses on the order of $1 \mu \mathrm{m},{ }^{10}$ such a

* To whom correspondence should be addressed. E-mail: carl.hagglund@ chalmers.se.

Received for review: $6 / 1 / 2010$

Published on Web: 00/00/0000 near-field approach is therefore well worth investigating in more detail.

In this Letter, we show that in ultrathin structures, where near-field effects dominate the response, the maximum light absorption is determined by a ratio of the external media refractive indices. The derived limit constitutes a benchmark for light absorption in nanoscale systems. We show that the optimal conditions can feasibly be realized in effectively zero thickness, two-dimensional (2D) nanocomposites of noble metals and solar cell materials. Such systems are found to have a highly interesting potential for solar cell applications, since more than $90 \%$ of the total absorption may be spatially located to the solar cell material thanks to an efficient exchange of oscillator strength with the plasmonic material. Nevertheless, the primary question addressed reaches beyond the field of solar cells. Namely, if relying on the nearfield interactions in ultrathin, nanostructured films, how high absorptance of light might be achieved in theory and for realistic systems, and how much of the absorbed energy can be channeled to a specific part of the structure? Apart from its fundamental interest, this question is also of high relevance for many optical sensing, photocatalytic, and photoelectrochemical applications.

The analysis of this Letter proceeds via three interconnected parts, where in the first two, the optimal conditions for light absorption in ultrathin homogeneous films and plasmonic nanoparticle arrays are investigated. In the third part, the effective medium properties of the different optimized systems are shown to be identical in the zerothickness/long wavelength limit.

We thus begin by addressing the question of how much light can ultimately be absorbed in structures where nearfield components dominate and effects depending on field 

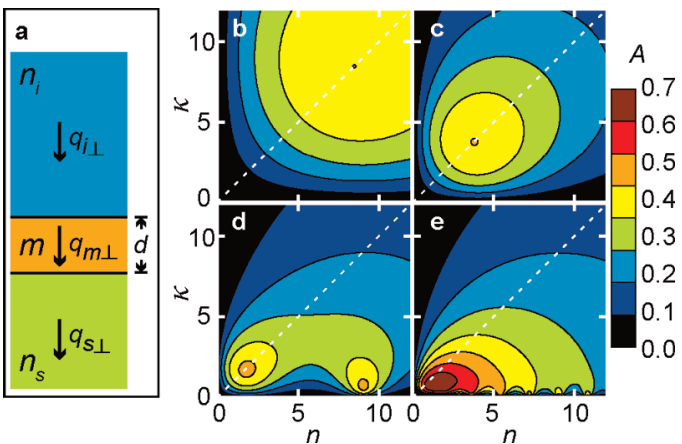

FIGURE 1. (a) Thin homogeneous film considered with complex refractive index $m=n+i \kappa$. The film is of thickness $d$ and embedded between two semi-infinite dielectrics of refractive indices $n_{\mathrm{i}}$ and $n_{\mathrm{s}}$, respectively. The incident electromagnetic plane wave has a vacuum wavenumber $q_{0}$ and wave vectors $\mathrm{q}_{\mathrm{i}}, \mathrm{q}_{\mathrm{m}}$ and $\mathrm{q}_{\mathrm{s}}$ (normal components $q_{\mathrm{i} \perp}, q_{\mathrm{m} \perp}$ and $\left.q_{\mathrm{s} \perp}\right)$ in the different media. In (b-e), absorptance in the film is shown as a function of its optical constants for normal incident light. The wavelength is $900 \mathrm{~nm}$, and the dielectric environment has $n_{\mathrm{i}}=n_{\mathrm{s}}=1$. The film thicknesses $d$ are $2,10,50$, and $250 \mathrm{~nm}$ in panels b, c, d, and e, respectively. The dotted lines mark $n=\kappa$ about which the shown absorption is nearly symmetric for the two thinnest films $(b, c)$. In these cases, the absorptance reaches a peak value of close to $50 \%$ on the symmetry line. For the two thicker films $(d, e)$, the peak height increases and additional maxima appear along the $n$-axis due to Fabry-Perot modes.

retardation are negligible. In such systems, consequences of thin film interference and coupling into guided and other propagating modes are insignificant. In practice, this is the case in structures where all characteristic dimensions are much smaller than the wavelength of light.

As a model system, we therefore consider a thin homogeneous film (thickness $d$ ) in the long wavelength limit (Figure $1 \mathrm{a}$ ). The complex refractive index $m=n+i \kappa$ of the film will be optimized for normal incident light having a vacuum wavelength $\lambda_{0}$ (wavenumber $q_{0}=2 \pi / \lambda_{0}$ ), and a small wavelength-normalized film thickness $\delta \equiv q_{0} d \ll 1$. In particular, the film thickness is required to be much smaller than the wavelength of light inside (and outside) the film, so that the wavelength-normalized optical path length $|m \delta| \ll 1$. Writing the reflection coefficients for transverse electric (TE) fields as $r_{x} \equiv\left(n_{x}-m\right) /\left(n_{x}+m\right)$ with $x=i, s$ for the front and back interfaces, respectively, the total reflection and transmission coefficients taking multiple internal reflections into account are ${ }^{11}$

$$
\tilde{r}=\frac{r_{\mathrm{i}}-r_{\mathrm{s}} \gamma^{2}}{1-r_{\mathrm{i}} r_{\mathrm{s}} \gamma^{2}} \text { and } \tilde{t}=\frac{\left(1+r_{\mathrm{i}}\right)\left(1-r_{\mathrm{s}}\right) \gamma}{1-r_{\mathrm{i}} r_{\mathrm{s}} \gamma^{2}} \exp \left(-i n_{\mathrm{s}} \delta\right)
$$

respectively, where $\gamma \equiv \exp (i m \delta)$. The thin film absorptance is achieved from

$$
A=1-|\tilde{r}|^{2}-C|\tilde{t}|^{2}
$$

where for TE-fields $C=n_{\mathrm{s}} / n_{\mathrm{i}}$ and for transverse magnetic (TM) fields $C=n_{\mathrm{i}} / n_{\mathrm{s}}$. Equations 1 and 2 are readily generalized to oblique incidence of the light by adopting the angular dependent Fresnel reflection coefficients, ${ }^{11}$ by including a trigonometric factor $q_{\mathrm{i}} q_{\mathrm{s} \perp} /\left(q_{\mathrm{i} \perp} q_{\mathrm{s}}\right)$ in $C$ that compensates for differing normal components of the energy fluxes to and from the film and by taking $\gamma \equiv \exp \left(i q_{\mathrm{m} \perp} d\right)$.

Figure $1 \mathrm{~b}-\mathrm{e}$ shows the absorptance from eq 2 as function of the optical constants of the film for four different film thicknesses. Local maxima of close to $50 \%$ absorptance are found close to the dotted lines $n=\kappa$, and additional maxima for higher values of $n$. The latter represent Fabry-Perot modes and occur for relatively small extinction coefficients $\kappa \ll n$. They approximately satisfy the condition for maximized transmission through a corresponding nonabsorbing layer, with $n \approx N \times \pi / \delta$ for $N=1,2,3 \ldots$ in vacuum. Clearly, only the "zeroth order" peak close to the line $n=\kappa$ is able to fulfill $|m \delta| \sim|n \delta| \ll 1$ in the limit of vanishing film thickness $(\delta \rightarrow 0)$, and we thus focus on the conditions for this particular optimum. From eq 1 , it is noted that if $|\mathrm{m}|$ is a finite number, the phase factor $\gamma \rightarrow 1$ as $\delta \rightarrow 0$. This results in $\tilde{r}=O(\delta), \tilde{t}=1+O(\delta)$ and a weak absorption $A=O(\delta)$. To go beyond this case, we consider the problem of how to choose an optimal complex refractive index of the film for a given, but small, film thickness. To fulfill $|m \delta| \ll 1$, the optimum must correspond to a large $|m| \sim \delta^{-v}$ with $0<v<$ 1. Such an optimum exists, and it is to leading orders in $\delta$ given by (see the Supporting Information),

$$
\begin{aligned}
& m_{\max }=n_{\max }+i \kappa_{\max }=\frac{(1+i)}{\sqrt{2}}\left(\frac{n_{\mathrm{i}}+n_{\mathrm{s}}}{\delta}\right)^{1 / 2}+ \\
& \frac{(1-i)}{6 \sqrt{2}} \frac{\left(n_{\mathrm{i}}^{3}+n_{\mathrm{s}}^{3}\right)}{\left(n_{\mathrm{i}}+n_{\mathrm{s}}\right)^{3 / 2}} \delta^{1 / 2}+O\left(\delta^{3 / 2}\right)
\end{aligned}
$$

Equation 3 confirms the observation from Figure 1 that $n \approx \kappa$ for this zeroth order maxima, and it further shows that optimal conditions generally cannot be met by sole adjustment of the film thickness $\delta$, as was previously suggested. ${ }^{12}$ Only for a film material of high and close to equal optical constants $(n \approx \kappa \gg 0)$, this would be a possibility. However, such a material would have to support a heavily damped bulk plasmon, which is not common at frequencies of visible or near-infrared light. The maximum thin film absorptance becomes, via the reflection and transmission coefficients associated with the optimum (see Supporting Information equations S3-S4)

$$
A_{\max }=\frac{n_{\mathrm{i}}}{n_{\mathrm{i}}+n_{\mathrm{s}}}\left[1+\frac{n_{\mathrm{s}}}{6}\left(3 n_{\mathrm{s}}-n_{\mathrm{i}}\right) \delta^{2}\right]+O\left(\delta^{4}\right)
$$

Figure $2 \mathrm{a}$ illustrates the thickness dependence given by eq 4. It compares very well with solutions obtained numeri- 


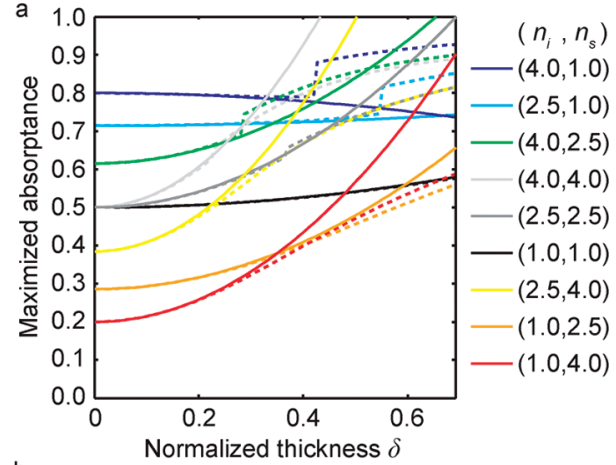

b

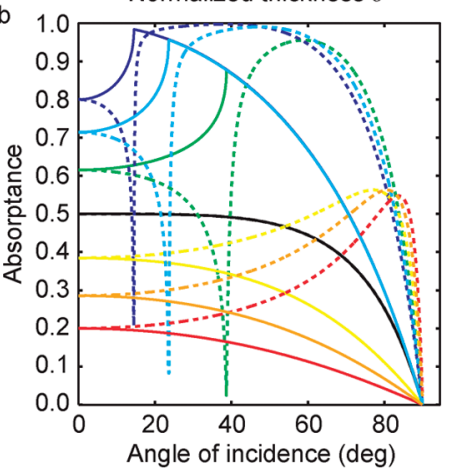

FIGURE 2. (a) Maximized absorptance as a function of normalized film thickness. The results were obtained numerically from the exact expressions (1) and (2) (dashed lines), and by means of the derived leading order approximation (4) (solid lines). Combinations of external media refractive indices $\left(n_{\mathrm{i}}, n_{\mathrm{s}}\right)$ are considered, as indicated to the right. The maximum $\delta$ plotted corresponds to a $100 \mathrm{~nm}$ thick film at a wavelength of $900 \mathrm{~nm}$. Abrupt jumps in the exact solutions are consequences of thin film interference. (b) Angular dependence of the thin film absorptance optimized for a small $\delta$ and normal incidence as in (a). The solid lines are for TE-polarized incident light, and the dashed for TM-polarized. There is a dip at the critical angle for TM-polarized light, but the response is otherwise high over a broad range of angles. The three symmetric cases with $n_{\mathrm{i}}=n_{\mathrm{s}}$ overlap.

cally using the exact eqs 1 and 2, and for instance reproduces an initially decreasing maximum absorptance with film thickness when $n_{\mathrm{i}}>3 n_{\mathrm{s}}$. The maximum possible absorptance is thus given by $n_{\mathrm{i}} /\left(n_{\mathrm{i}}+n_{\mathrm{s}}\right)$ in the limit of vanishing film thickness in line with what was recently reported elsewhere. ${ }^{12,13}$ The explicit weak dependence on film thickness $\left(\propto \delta^{2}\right)$ derived here supports the importance of this limit for the achievable visible light absorption in nanoscale systems, for which it provides a useful benchmark. Further, Figure $2 \mathrm{~b}$ demonstrates a generally favorable angular dependence for films optimized according to eq 3, and thus a promising potential for practical applications also when light is incident from an angle. On the other hand, we have already noted that simple materials generally are not suitable for this purpose. We therefore go on to investigate nanocomposites, for which the effective optical properties can be tuned.

In the second part of this Letter we show that the identified zeroth order optimum for light absorption may indeed be satisfied by combining different materials into thin nanocomposite layers. The particular situation where core/

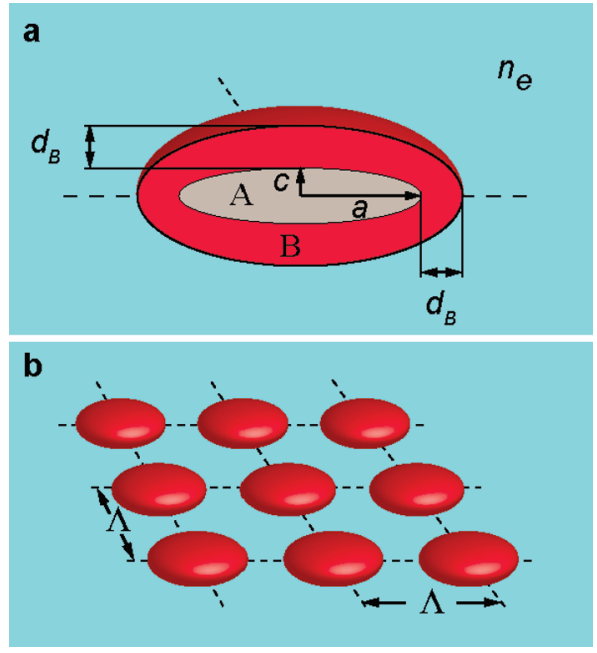

FIGURE 3. Investigated 2D array of oblate core/shell ellipsoids. (a) Cross section of a core/shell particle constituting the basic element of the array illustrated in (b). The core (A) and shell (B) are characterized by their dielectric functions $\varepsilon_{\mathrm{A}}$ and $\varepsilon_{\mathrm{B}}$, respectively, and assumed to be nonmagnetic. The external medium has a real refractive index $n_{\mathrm{e}}$. The two core semiaxes in the lattice plane have an equal length $a$, and the semiaxis normal to this plane has length $c$. The shell adds a thickness $d_{\mathrm{B}}$ in the directions of these axes. The core volume is characterized by its equivalent sphere radius, $r_{\mathrm{o}} \equiv$ $\left(3 V_{\mathrm{A}} / 4 \pi\right)^{1 / 3}$. The normal incident plane wave has its fields aligned with the lattice vectors, and the lattice constants are $\Lambda$ in both directions.

shell nanoparticles are arranged in a square 2D array, illustrated in Figure 3, is primarily considered. The core/shell system is useful for understanding the plasmon near-field induced part of the response in many device configurations ${ }^{5}$ and comes reasonably close to some experimental systems recently investigated. ${ }^{3}$ It is thus feasible to implement and serves as a guide for such experiments. Furthermore, the coupling between the constituent materials is nearly ideal in this type of structures. ${ }^{5}$ A desired exchange of oscillator strength between the core and shell may therefore take place efficiently, analogous to that between atomic core and valence electrons. ${ }^{14}$ We analyze a model with respect to maximum absorption of normal incident light in the long wavelength limit and then compare the results to numerical calculations by the finite element method (FEM).

We consider ellipsoidal core/shell particles with shape dependent depolarization factors ${ }^{11} L_{\mathrm{A}}$ and $L_{\mathrm{B}}$ for the core (A) and shell (B), respectively. The particle shape is generally a powerful parameter for tuning resonances to desired spectral positions. In terms of the ratio of the core volume $V_{\mathrm{A}}$ to the total volume $V_{\mathrm{AB}}, K \equiv V_{\mathrm{A}} / V_{\mathrm{AB}}$, the particle polarizability $\alpha_{A B}$ normalized by $3 V_{A B} / 4 \pi$ becomes ${ }^{11}$

$$
\bar{\alpha}_{\mathrm{AB}} \equiv \frac{\left(\varepsilon_{\mathrm{B}}-\varepsilon_{\mathrm{e}}\right)\left[\varepsilon_{\mathrm{B}}+\left(\varepsilon_{\mathrm{A}}-\varepsilon_{\mathrm{B}}\right) L_{\mathrm{AB}}\right]+K \varepsilon_{\mathrm{B}}\left(\varepsilon_{\mathrm{A}}-\varepsilon_{\mathrm{B}}\right)}{3\left\{\left[\varepsilon_{\mathrm{B}}+\left(\varepsilon_{\mathrm{A}}-\varepsilon_{\mathrm{B}}\right) L_{\mathrm{AB}}\right]\left[\varepsilon_{\mathrm{e}}+\left(\varepsilon_{\mathrm{B}}-\varepsilon_{\mathrm{e}}\right) L_{\mathrm{B}}\right]+K L_{\mathrm{B}} \varepsilon_{\mathrm{B}}\left(\varepsilon_{\mathrm{A}}-\varepsilon_{\mathrm{B}}\right)\right\}}
$$


where a volume-weighted combination of core and shell depolarization factors was introduced as $L_{\mathrm{AB}} \equiv L_{\mathrm{A}}-K L_{\mathrm{B}}$. Given the polarizability $\bar{\alpha}_{A B}$ of the basic building block, it has earlier been shown ${ }^{15}$ that absorption of normal incident light in a 2D square lattice of dipoles, using our notation above, is

$$
A=\frac{2 \operatorname{Im} \bar{U} \operatorname{Im} \bar{\alpha}_{\mathrm{AB}}}{\left|1-\bar{U} \bar{\alpha}_{\mathrm{AB}}\right|^{2}}
$$

The polarizability renormalization factor $\bar{U}$ due to the interaction between the particles is given by

$$
\bar{U} \equiv f+i F=1.08 V_{\mathrm{AB}} / \Lambda^{3}+3 i_{\mathrm{e}} q_{0} V_{\mathrm{AB}} / 2 \Lambda^{2}
$$

to lowest orders in $\Lambda / \lambda$. The factor 1.08 in the real part $f$ is the Topping factor, which varies only slightly with the lattice type. $^{16}$

As shown in the Supporting Information, the array absorptance peaks at $1 / 2$ when

$$
\bar{U}^{*} \bar{\alpha}_{\mathrm{AB}}=1
$$

This resonance condition may generally be applied to particles optically well described by a dipole polarizability $\bar{\alpha}_{\mathrm{AB}}$, and it can, for instance, be used to evaluate which type of materials that may fulfill optimal conditions for absorption in these types of arrays. For the particular case of core/shell particles, condition 8 gives two plasmonic eigenmodes, which may be expressed in terms of the shell dielectric function $\varepsilon_{B}$ as

$$
\varepsilon_{\mathrm{B}}^{\max } / \varepsilon_{\mathrm{e}}=Q\left(\chi_{\text {eff }}\right) \pm \sqrt{Q^{2}\left(\chi_{\text {eff }}\right)+P^{2}\left(\chi_{\text {eff }}\right)}
$$

where $\chi_{\text {eff }} \equiv 1-1 / L_{\text {eff }}$ and the effective depolarization factor for the shell is $L_{\text {eff }} \equiv L_{\mathrm{B}}-\bar{U}^{*} / 3$. Further, $P^{2}(\chi)=\chi L_{\mathrm{AB}} \varepsilon_{\mathrm{A}} / \varepsilon_{\mathrm{e}} /$ $\left(1-L_{\mathrm{K}}\right)$ with $L_{\mathrm{K}} \equiv L_{\mathrm{AB}}+K$, and $Q(\chi)=\left[\left(1-L_{\mathrm{AB}}\right) \chi-\right.$ $\left.L_{K} \varepsilon_{A} / \varepsilon_{e}\right] /\left[2\left(1-L_{K}\right)\right]$. The two solutions in eq 9 correspond to an inner, core localized surface plasmon mode (positive sign), and an outer, shell localized surface plasmon mode (negative sign), respectively. The shell plasmon mode requires a metallic shell $\left(\kappa_{\mathrm{B}}>n_{\mathrm{B}}\right)$, while the core plasmon mode occurs for a nonmetallic shell $\left(\kappa_{\mathrm{B}}<n_{\mathrm{B}}\right)$ and a metallic core. Illustrations of the dependence on shell optical constants are provided in Figure $4 \mathrm{a}-\mathrm{d}$, where the total absorptance at $\lambda_{0}$ $=900 \mathrm{~nm}$ is plotted for the case of an Ag-core ${ }^{17}$ and four different lattice constants of the 2D array. The two absorptance maxima are clearly distinguished and accurately

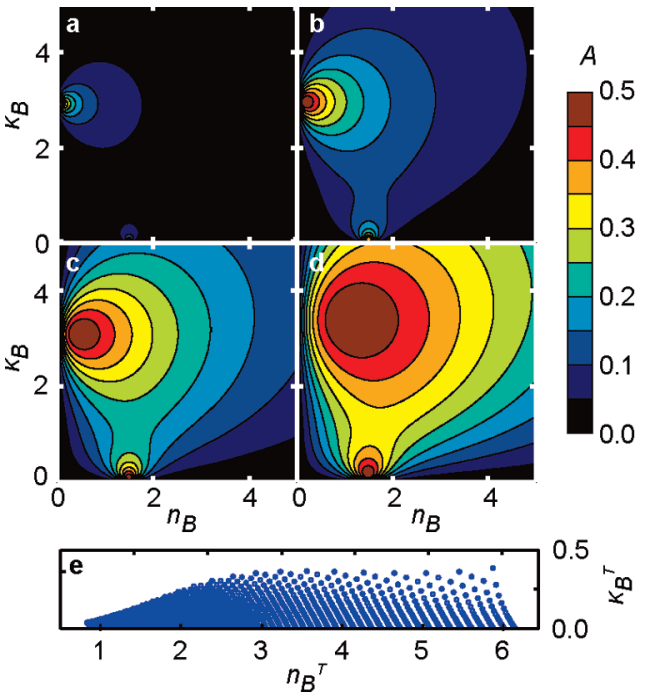

FIGURE 4. $(a-d)$ Absorptance as function of shell optical constants in core/shell nanoparticle arrays, at a wavelength $\lambda_{0}=900 \mathrm{~nm}$ and different lattice constants. The core had the optical constants of Ag, ${ }^{17}$ and a sphere equivalent core radius $r_{\mathrm{o}}=18.3 \mathrm{~nm}$. The shell thickness $d_{\mathrm{B}}=10 \mathrm{~nm}$ and the external medium $n_{\mathrm{e}}=1.5$. Two maxima are always observed, with amplitudes of $1 / 2$. These correspond to a core plasmon mode $\left(n_{\mathrm{B}}>\kappa_{\mathrm{B}}\right)$ and a shell plasmon mode $\left(\kappa_{\mathrm{B}}>n_{\mathrm{B}}\right)$, respectively. The particle shapes (core aspect ratio a/c) were determined such that the core plasmon mode was located on the line $n_{\mathrm{B}}=n_{\mathrm{e}}=1.5$ for lattice constants $\Lambda$ of (a) 480, (b) 250 , (c) 160 and (d) $110 \mathrm{~nm}$, respectively. In the scatter plot (e), each point represents an optimal solution for the shell optical constants for the core plasmon mode with Ag cores. The conditions are again $n_{\mathrm{e}}=$ $1.5, d_{\mathrm{B}}=10$ and $r_{\mathrm{o}}=18.3 \mathrm{~nm}$. The semiaxes lengths were varied within a limited, feasible range for nontouching, oblate particles $\left[\left(3 V_{\mathrm{A}} / 4 \pi\right)^{1 / 3}<a<\Lambda / 2-d_{\mathrm{B}}\right.$ and $\left.c>2 \mathrm{~nm}\right]$ while the lattice constants were limited to $30 \mathrm{~nm}<\Lambda<\lambda_{0} / 2$.

predicted by eq 9 . The parameters of Figure 4 were chosen for ease of comparison with previous FEM calculations ${ }^{5}$ with good agreement found; at the core plasmon peak, the differences are within $10 \%$ of the optimal parameter values of $a$ and $\kappa_{\mathrm{B}}$. The most sparse array is shown in Figure 4a, and results in a core plasmon resonance being realized for approximately uncoated Ag cores, that is, $n_{\mathrm{B}}^{\max }=n_{\mathrm{e}}$ and $\kappa_{\mathrm{B}}^{\max }$ $\approx 0$. For the denser arrays of Figure $4 b-d$, the optima broaden. Such resonance peak broadening is a well-known effect of increased damping in the system. The maximizing damping part of the permittivity ( $\operatorname{Im} \varepsilon_{\mathrm{B}}=2 n_{\mathrm{B}} \kappa_{\mathrm{B}}$ ) is under the investigated conditions closely related to the array density, such that $\kappa_{\mathrm{B}}^{\max } \propto \Lambda^{-2}$.

In the scatter plot of Figure 4e, each point represents a realistic solution for the core plasmon mode. The extensive spread shows that many absorber materials of interest for thin film photovoltaics (such as CIS/CIGS, ${ }^{18} \mathrm{a}$-Si, ${ }^{19}$ organic semiconductors ${ }^{20}$ and dye molecules ${ }^{5}$ ) can be matched, for which typically $1.5<n_{\mathrm{B}}<5$ and $10^{-2}<\kappa_{\mathrm{B}}<1$ in the visible to near-infrared range. This spread results from a realistic variation of $\Lambda$ and only oblate aspect ratios $(a / c>1$ ), leaving several important parameters (especially shell thickness and particle volume) free for a practical system design. When also including prolate shapes $(a / c<1)$, similar results are 


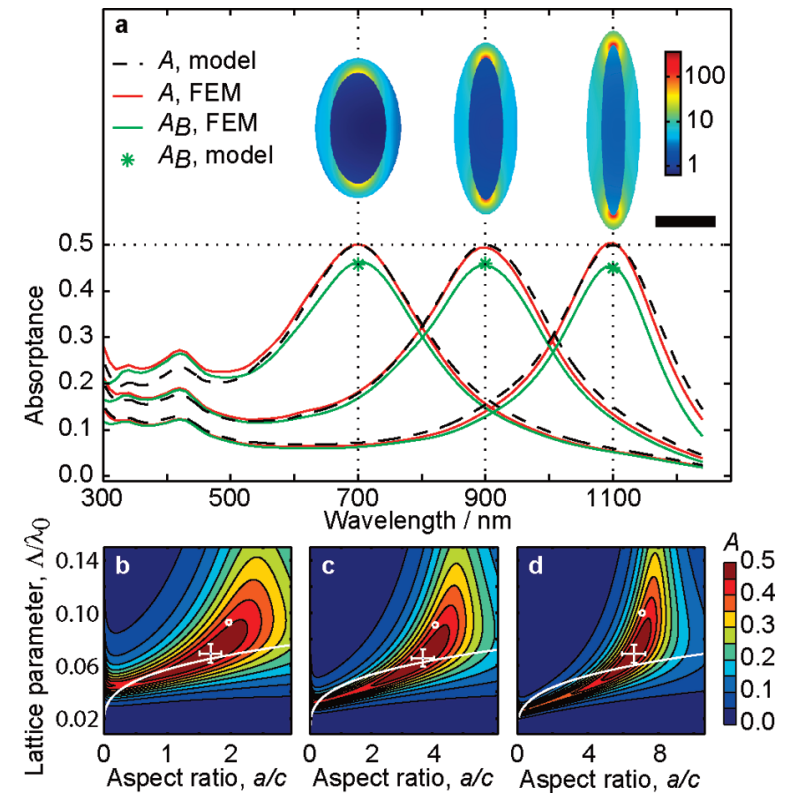

FIGURE 5. (a) Absorptance maximized for wavelengths of $\lambda_{0}=700$, 900 , and $1100 \mathrm{~nm}$, obtained using the analytic model and FEM. The considered 2D arrays are located in a medium where $n_{\mathrm{e}}=2$, and consist of Ag-core/CIS-shell particles with $d_{\mathrm{B}}=5 \mathrm{~nm}$ and $r_{\mathrm{o}}=15$ $\mathrm{nm}$. The core aspect ratios and normalized lattice constants were optimized to maximize the total absorptance $A$ at $\lambda_{0}$. The shell absorptance $A_{\mathrm{B}}$ constitutes a high fraction of $A$ across the entire spectrum, according to FEM. The model estimate of $A_{\mathrm{B}}$ is also included at $\lambda_{0}$. Above each peak, the local absorption rates are displayed in cross sections of the corresponding particles. In these FEM plots, vertical polarized light was incident from the left. The color scale is normalized and the scale bar $20 \mathrm{~nm}$. In $(b-d)$, the model absorptance is shown as a function of the geometric parameters that were optimized in (a) for the targeted wavelengths $\lambda_{0}=$ $700 \mathrm{~nm}$ in (b), $900 \mathrm{~nm}$ in (c) and $1100 \mathrm{~nm}$ in (d), respectively. The optima, marked by the white crosses, are robust in the sense that the absorptance is typically reduced by less than $10 \%$ (from 50 to $45 \%$ ) for $\pm 10 \%$ variations of the underlying parameters (indicated by the cross size in each direction). For comparison, the optima identified in the corresponding FEM calculations are shown by white circles. Solutions are not valid below the white curves, where particles overlap.

obtained for most parts of the visible to near-infrared range. Optimal conditions may thus be realized for an extensive range of practically achievable parameters.

The wavelength dependence of these kind of optimized arrays are presented in Figure $5 \mathrm{a}$, for the more concrete case of Ag-cores ${ }^{17}$ with CuInSe ${ }_{2}$ (CIS)-shells. ${ }^{18}$ Excellent agreement of the absorptance is found for structures optimized according to the analytic model, and by use of FEM calculations according to previously published methods. ${ }^{21,22}$ On the other hand, Figure $5 b-d$ shows a systematic offset in the specific optimal parameters identified by the two methods. This may qualitatively be understood as a consequence of taking only dipolar contributions into account in the analytic model, whereas the finite size of the particles are taken into full account in the FEM calculations. In the latter case, field retardation across the particles is present, and results in coupling to higher order modes (quadrupolar, octupolar, etc.). ${ }^{11}$ This introduces additional damping channels in the system (which lowers $\kappa_{\mathrm{B}}^{\max }$ ), so that the more realistic FEM calculations give an optimum for sparser arrays (since $\Lambda \sim$ $\left.\left(\kappa_{\mathrm{B}}^{\max }\right)^{-1 / 2}\right)$. Apart from this offset, the analytic model is a very useful tool for identifying and understanding trends, and mappings such as Figure $5 b-d$ provide good estimates of the widths and sensitivities of the optimum to changes of various conditions. The wavelength dependences of Figure $5 a$ are good examples of this. We note that the relatively high damping in the CIS/Ag system leads to robust optima that are much more feasible to realize in practice compared to, for instance, uncoated Ag nanoparticle arrays (as in Figure 4a). It is also noteworthy that the optimal conditions identified by FEM correspond to Ag- and CIS-thicknesses (volumes per surface area) of only 4 and $5 \mathrm{~nm}$, respectively. This is 3 orders of magnitude lower than in typical thin film solar cells, and since the peak width is appreciable, this result indicates a high potential for saving materials and resources in such applications.

For this concept to be useful in photovoltaics and other applications, a high fraction of the absorption must typically (but not necessarily ${ }^{23,24}$ ) be localized to the semiconductor rather than to the metal parts. As long as the particles behave as point dipoles, the derived maximum absorptance will remain the same regardless of materials. However, the internal spatial distribution of the absorbed energy will depend on the particle constituents and their detailed arrangement. An analytic estimate of this distribution is therefore of interest, and can be achieved by comparison with an artificial situation where the damping in the metal part is suppressed. Because the local absorption rate is, for nonmagnetic media, directly proportional to the damping part of the permittivity, it is plausible to assume that the absorbed fractions will distribute in proportion to this value. For the core/shell case, this implies a core absorptance $A_{\mathrm{A}}$ $\propto \operatorname{Im} \varepsilon_{\mathrm{A}}$ and a shell absorptance $A_{\mathrm{B}} \propto \operatorname{Im} \varepsilon_{\mathrm{B}}$, respectively. If the total absorptance is maximized (to $1 / 2$ ), the shell absorptance is then $A_{\mathrm{B}}=\operatorname{Im} \varepsilon_{\mathrm{B}}^{\max } /\left(2 \operatorname{Im} \varepsilon_{\mathrm{B}}^{0}\right)$, where $\varepsilon_{\mathrm{B}}^{\max }$ is the maximizing shell permittivity from eq 9 , and $\varepsilon_{\mathrm{B}}^{0}$ is the particular value of $\varepsilon_{\mathrm{B}}^{\max }$ when $\operatorname{Im} \varepsilon_{\mathrm{A}}=0$. The corresponding branching ratio for shell absorption is $\Gamma_{\mathrm{B}} \equiv A_{\mathrm{B}} / A=\mathrm{Im}$ $\varepsilon_{\mathrm{B}}^{\max } / \operatorname{Im} \varepsilon_{\mathrm{B}}^{0}$. These estimates and their underlying assumption is well supported by the good agreement found with the independent FEM calculations shown in Figure $5 \mathrm{a}$ and 6. Figure 6 further illustrates that higher branching ratios are attainable in denser arrays. This is again understood from an absorption distribution proportional to the damping of the materials, and the observed scaling $\operatorname{Im} \varepsilon_{\mathrm{B}}^{\max } \propto \kappa_{\mathrm{B}}^{\max } \propto \Lambda^{-2}$. It is encouraging that systems optimized for $\mathrm{Ag}$ and common, high-damping solar cell materials (Im $\left.\varepsilon_{B} \sim 1\right)^{18-20}$ may have branching ratios for semiconductor absorption in excess of $90 \%$. Such high-damping configurations have the additional advantage of resulting in a broader response and thus better coverage of the solar spectrum. It is also noteworthy that a maximization of the semiconductor absorptance alone rather than the total absorptance, would result 


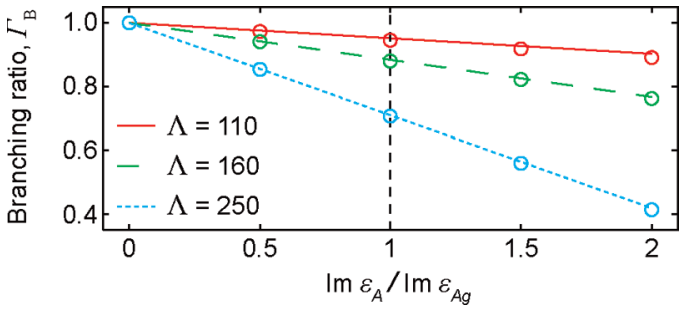

FIGURE 6. Model (lines) and FEM $(O)$ calculated branching ratios for shell absorption. The particle cores have a permittivity real part as for $\mathrm{Ag},{ }^{17}$ but varied damping part (Im $\left.\varepsilon_{\mathrm{A}}\right)$. The sphere equivalent core radius was $r_{\mathrm{o}}=18.3 \mathrm{~nm}$ and the shell thickness $d_{\mathrm{B}}=10 \mathrm{~nm}$. For the lattice constants indicated in nm units, $\varepsilon_{\mathrm{B}}$ was optimized for $\lambda_{0}$ $=900$ and $n_{\mathrm{e}}=1.5$. For each value of the lattice constants, the particle shapes were determined such that $n_{\mathrm{B}}=1.5$ for an Ag core (vertical line).

in yet higher branching ratios. Altogether, these results demonstrate the feasibility of a high quantum efficiency in solar cells based on plasmonic near-field effects.

In the final part of this Letter, we note that the optimal core/shell arrays generate, in an effective sense, the very same conditions as those observed for ultrathin homogeneous films at maximum absorptance. This is proven by representing the array by a thin film having a single effective medium permittivity and thickness, as detailed in the Supporting Information. Specifically, the array resonance condition (8) produces effective optical constants as for an ultrathin film optimized according to eq 3. Because the resonance condition (8) applies to any particles in the long wavelength limit, there is no unique feature of the core/shell array leading to this coincidence. Rather, this is a manifestation of the fact that many different types of nanostructures, and especially plasmonic nanocomposites, can fulfill the same optimal conditions in this effective sense. Metal nanoparticle $^{25}$ or cylinder ${ }^{26}$ arrays placed on or within a thin, continuous semiconductor film ${ }^{5}$ are important examples of this from a practical view. Regarding the effective thickness of the array, it is sometimes taken to equal the particle height. ${ }^{27}$ Others have introduced a fictitious volume fraction and from the Maxwell-Garnett expression inferred a film thickness equal to the lattice constant $\Lambda .{ }^{15}$ However, since from eq 6 the maximum absorptance in the particle array is exactly one-half, eq 4 implies that this thickness is in fact zero. Just as small particles may often be treated as point (OD) dipoles, an array of such particles behaves as a truly $2 \mathrm{D}$ system. The FEM calculations show that this 2D model works as a good approximation to arrays of 3D particles of practical interest. An important implication is that the maximum dipolar array absorptance is $A=n_{\mathrm{i}} /\left(n_{\mathrm{i}}+n_{\mathrm{s}}\right)$ in the more general case when $n_{\mathrm{i}} \neq n_{\mathrm{s}}$. This relation provides an opportunity for increasing the maximum absorption by design of the dielectric environment. In a similar way, yet higher levels of absorption may be accomplished by addition of antireflective or reflective layers to the system (see the Supporting Information). ${ }^{28}$ As a third route to increase (and broaden) the absorptance, higher order multipolar particle modes may be exploited. ${ }^{2}$ We finally note that the angular response of arrays is somewhat more complicated than in films, for instance due to anisotropic particle polarizabilities and more elaborate coupling dependencies in the array. However, the basic physics involved is the same, and very attractive results have been demonstrated in related systems at oblique angles. ${ }^{26}$

To conclude, we have demonstrated a "zero order" optimum for absorption of light in ultrathin nanostructured films, based on near-field coupling between the constituents. The associated maximum absorptance is determined by the external media refractive indices and given by $A=n_{\mathrm{i}} /\left(n_{\mathrm{i}}+\right.$ $\left.n_{\mathrm{s}}\right)+O\left(d^{2} / \lambda^{2}\right)$. This limit constitutes a general benchmark for light absorption in nanoscale systems extended in two dimensions. It can be realized in plasmonic nanoparticle arrays which, in the long wavelength limit, are shown to behave as truly two-dimensional structures of zero thickness. Expressions were supplied and shown useful for analytic determination of the optimal conditions in core/shell nanoparticle arrays. By combining low-damping metals (such as Ag) and common, high-damping thin film solar cell materials (such as a-Si, CIS, CIGS, or organic semiconductors), more than $90 \%$ of the absorbed radiation can be channeled to the solar cell material. These plasmonic nanocomposite systems thus show great promise for the development of, among other things, cheap and efficient photovoltaics.

Acknowledgment. C.H. and B.K. acknowledge funding from the interconnected projects "Nanotechnology for Sustainable Energy" from the Chalmers Foundation and "NanoSEE" from the Swedish Energy Agency.

Supporting Information Available. Ultimate thickness limit for efficient solar cells; condition for maximum absorption in ultrathin films; condition for maximum absorption in two-dimensional particle arrays; use of antireflective and reflective layers; and effective medium model for nanoparticle arrays. This material is available free of charge via the Internet at http://pubs.acs.org.

\section{REFERENCES AND NOTES}

(1) Hallermann, F.; Rockstuhl, C.; Fahr, S.; Seifert, G.; Wackerow, S.; Graener, H.; von Plessen, G.; Lederer, F. Phys. Status Solidi A 2008, 205 (12), 2844-2861.

(2) Akimov, Y. A.; Koh, W. S.; Ostrikov, K. Opt. Express 2009, 17 (12), 10195-10205.

(3) Standridge, S. D.; Schatz, G. C.; Hupp, J. T.J. Am. Chem. Soc. 2009, 131 (24), 8407-8409.

(4) Catchpole, K. R.; Polman, A. Appl. Phys. Lett. 2008, 93 (19), 191113

(5) Hägglund, C.; Kasemo, B. Opt. Express 2009, 17 (14), 11944-11957.

(6) Pala, R. A.; White, J.; Barnard, E.; Liu, J.; Brongersma, M. L. Adv. Mater. 2009, 21 (34), 3504

(7) Catchpole, K. R.; Polman, A. Opt. Express 2008, 16 (26), $21793-$ 21800 .

(8) Atwater, H. A.; Polman, A. Nat. Mater. 2010, 9 (3), 205-213.

(9) Rand, B. P.; Peumans, P.; Forrest, S. R. J. Appl. Phys. 2004, 96 (12), 7519-7526.

(10) Markvart, T.; Castaner, L. Practical handbook of photovoltaics: fundamentals and applications; Elsevier Advanced Technology: New York, 2003; p 984. 
(11) Bohren, C. F.; Huffman, D. R. Absorption and Scattering of Light by Small Particles; Wiley-VCH: Weinheim, 2004.

(12) Driessen, E. F. C.; Braakman, F. R.; Reiger, E. M.; Dorenbos, S. N.; Zwiller, V.; de Dood, M. J. A. Eur. Phys. J. 2009, 47 (1), 10701.

(13) Driessen, E. F. C.; de Dood, M. J. A. Appl. Phys. Lett. 2009, 94 (17), 171109

(14) Smith, D. Y. Dispersion theory, sum rules, and their application to the analysis of optical data. In Handbook of Optical Constants of Solids; Edward, D. P., Ed.; Academic Press: Burlington, 1997.

(15) Vlieger, J. Physica 1973, 64 (1), 63-81.

(16) Topping, J. Proc. R. Soc. London 1927, 114, 67-72.

(17) Johnson, P. B.; Christy, R. W. Phys. Rev. B 1972, 6 (12), 4370 4379 .

(18) Alonso, M. I.; Wakita, K.; Pascual, J.; Garriga, M.; Yamamoto, N. Phys. Rev. B 2001, 63 (7), No. 075203.

(19) Piller, H. Amorphous Silicon. In Handbook of Optical Constants of Solids; Palik, E. D., Ed.; Academic Press: New York, 1985; p 571
(20) Hoppe, H.; Sariciftci, N. S.; Meissner, D. Mol. Cryst. Liq. Cryst. 2002, 385, 233-239.

(21) Hägglund, C.; Zäch, M.; Petersson, G.; Kasemo, B. Appl. Phys. Lett. 2008, 92 (5), No. 053110

(22) Dmitriev, A.; Hägglund, C.; Chen, S.; Fredriksson, H.; Pakizeh, T.; Käll, M.; Sutherland, D. S. Nano Lett. 2008, 8 (11), 3893-3898.

(23) Westphalen, M.; Kreibig, U.; Rostalski, J.; Luth, H.; Meissner, D. Sol. Energy Mater. Sol. Cells 2000, 61 (1), 97-105.

(24) Moulin, E.; Luo, P. Q.; Pieters, B.; Sukmanowski, J.; Kirchhoff, J.; Reetz, W.; Muller, T.; Carius, R.; Royer, F. X.; Stiebig, H. Appl. Phys. Lett. 2009, 95 (3), No. 033505.

(25) de Abajo, F. J. G. Rev. Mod. Phys. 2007, 79 (4), 1267-1290.

(26) Laroche, M.; Albaladejo, S.; Gomez-Medina, R.; Saenz, J. J. Phys. Rev. B 2006, 74 (24), 245422.

(27) Barrera, R. G.; del Castillo-Mussot, M.; Monsivais, G.; Villaseñor, P.; Mochan, W. L. Phys. Rev. B 1991, 43 (17), 13819-13826.

(28) Hägglund, C.; Apell, S. P. Opt. Express 2010, 18 (103), to be published. 